\title{
Pathogenic variant in NLRP7 (19q13.42) associated with recurrent gestational trophoblastic disease: Data from early embryo development observed during in vitro fertilization
}

\author{
E. Scott Sills ${ }^{1,2}$, Alexandra J. Obregon-Tito ${ }^{3}$, Harry Gao ${ }^{3}$, Thomas K. McWilliams ${ }^{4}$, Anthony T. Gordon ${ }^{4}$, Catharine A. Adams ${ }^{5}$, \\ Rima Slim 6 \\ 'Reproductive Research Section, Center for Advanced Genetics, Carlsbad, CA; ${ }^{2}$ Department of Obstetrics and Gynecology, Palomar Medical Center, \\ Escondido, CA; ${ }^{3}$ Fulgent Diagnostics, Temple City, CA; ${ }^{4}$ Genesis Genetics Inc., Plymouth, Ml; ${ }^{5}$ Reproductive Sciences Medical Center, San Diego, CA, USA; \\ ${ }^{6}$ Department of Human Genetics, McGill University Health Centre Research Institute, Montréal, QC, Canada
}

Objective: To describe in vitro development of human embryos derived from an individual with a homozygous pathogenic variant in NLRP7 (19q13.42) and recurrent hydatidiform mole (HM), an autosomal recessive condition thought to occur secondary to an oocyte defect.

Methods: A patient with five consecutive HM pregnancies was genomically evaluated via next generation sequencing followed by controlled ovarian hyperstimulation, in vitro fertilization (IVF) with intracytoplasmic sperm injection, embryo culture, and preimplantation genetic screening. Findings in NLRP7 were recorded and embryo culture and biopsy data were tabulated as a function of parental origin for any identified ploidy error.

Results: The patient was found to have a pathogenic variant in NLRP7 (c.2810+2T $>\mathrm{G})$ in a homozygous state. Fifteen oocytes were retrieved and 10 embryos were available after fertilization via intracytoplasmic sperm injection. Developmental arrest was noted for all 10 embryos after 144 hours in culture, thus no transfer was possible. These non-viable embryos were evaluated by karyomapping and all were diploid biparental; two were euploid and eight had various aneuploidies all of maternal origin.

Conclusion: This is the first report of early human embryo development from a patient with any NLRP7 mutation. The pathogenic variant identified here resulted in global developmental arrest at or before blastocyst stage. Standard IVF should therefore be discouraged for such patients, who instead need to consider oocyte (or embryo) donation with IVF as preferred clinical methods to treat infertility.

Keywords: Hydatidiform mole; In vitro fertilization; Preimplantation embryo development; Recurrent miscarriage; Reproductive genetics

\section{Introduction}

Hydatidiform mole (HM) is sporadic and androgenetic diploid in origin, and represents the most common type of gestational tropho-

Received: Nov 7, 2016 · Revised: Dec 1, 2016 · Accepted: Feb 28, 2017

Corresponding author: E. Scott Sills

Reproductive Research Section, Center for Advanced Genetics, 3144 El Camino Real, Suite 106, Carlsbad, CA 92008, USA

Tel:+1-760-994-0156 Fax:+1-760-994-0159 E-mail:drsills@CAGivf.com

This is an Open Access article distributed under the terms of the Creative Commons Attribution Non-Commercial License (http://creativecommons.org/licenses/by-nc/4.0/) which permits unrestricted non-commercial use, distribution, and reproduction in any medium, provided the original work is properly cited. blastic disease. These pathologic conceptions are thought to result from fertilization of an oocyte where maternal chromosomes have been lost and endoreduplication of a single sperm genome occurs (or fertilization by two sperm), yielding a diploid DNA content derived entirely from the paternal genome [1]. Rarely, $\mathrm{HM}$ can be recurrent or familial [2,3]. Detailed homozygosity mapping and gene mutation screening in these settings has identified two causative loci, 19q13.4 and 6q13-chromosomal regions which contain NLRP7 and KHDC3L, respectively [4]. Of note, approximately $70 \%$ of women affected by familial recurrent HM have autosomal recessive mutations of NLRP7 [5]. Compelling evidence that an abnormal oocyte is re- 
sponsible for the pathophysiology of recurrent HM is provided from observations that in vitro fertilization (IVF) cycles using donated oocytes for women with NLRP7 mutations result in normal offspring [6]. Nevertheless, several questions remain open regarding this phenomenon: (1) is the NLRP7 protein and/or its corresponding ribonucleic acid (RNA) required in the oocyte, and, if yes, at what stages; (2) what are the consequences of recessive NLRP7 mutations for the oocyte and for the embryo (cultured in vitro) which derives from it; and (3) when do embryo developmental defects manifest in this setting? This case report contributes new information addressing each of these unresolved issues.

\section{Methods}

\section{Clinical presentation and genetic assessment}

A 35-year-old G5P0050 presented with her husband for reproductive endocrinology consultation. Both partners were of Latino heritage and in excellent health. Her blood type/Rh group was A positive; she had no known drug allergies; and the patient took no medication on a regular basis. Between 2001 and 2012, the couple established five unassisted conceptions, all of which were HM and electively terminated in the first trimester. After the initial pregnancy, additional evaluation discovered that the patient had an European Society of Human Reproduction and Embryology Class Ilb uterine anomaly/bicornuate uterus although her medical records did not define which uterine compartment contained the pregnancy for all cases. To evacuate all molar tissue, each event was followed by dilation and curettage performed by a gynecologist in an outpatient setting. However for her most recent pregnancy in 2012, serum human chorionic gonadotropin ( $\mathrm{hCG}$ ) levels did not decline normally after surgery and the patient received nine cycles of intravenous. doxorubicin [7] for low risk gestational trophoblastic disease under co-management with an oncology team. She was then placed on oral contraceptive pills, referred to a genetic counselor and advised not to conceive again for 12 months.

\section{Molecular characterization of parents at NLRP7}

The couple expressed interest in IVF and preimplantation genetic screening (PGS) of embryos before transfer. Anonymous donor oocyte IVF was also discussed but this option was declined as first-line treatment for personal reasons. While basic chromosomal data (standard G-band karyotypes) from both partners had been reassuring, further evaluation and counseling was obtained before moving forward with IVF in April 2016. Written informed consent for clinical and research aspects of the proposed treatment were obtained from both partners, and the investigational component was approved by our laboratory's independent institutional review board. The rare na- ture of recurrent $\mathrm{HM}[3,8]$ was discussed and genomic sequencing for the NLRP7 gene was carried out for both individuals. Briefly, genomic DNA was extracted from blood samples collected by peripheral venipuncture and, after evaluating the quantity and quality of the DNA, nucleic acids were disrupted to mean fragment size of 250 bp. Hybrid capture was used to enrich for areas of interest that included the coding and splicing regions of the NLRP7 gene. Pair-ended DNA sequences were obtained by next generation sequencing (NGS) using the NextSeq platform (Illumina, San Diego, CA, USA) at a mean depth of 100X; all regions of interest were characterized by coverage equal to or greater than 20X. Analysis of deletions and duplications was derived from NGS data; potential deletions or duplications were excluded for the index case.

\section{Follicular recruitment, IVF protocol, and fertilization technique}

Pre-IVF screening tests were normal for both partners. Ovarian reserve [9] was considered satisfactory by serum anti-Müllerian hormone and antral follicle count estimates ( $4.63 \mathrm{ng} / \mathrm{mL}$ and $\mathrm{n}=12$, respectively). Monophasic oral contraceptive pills were used for suppression in the cycle immediately prior to IVF. No ovarian cysts $>15$ $\mathrm{mm}$ diameter were detected and baseline serum estradiol was $<80$ $\mathrm{pg} / \mathrm{mL}$. The patient received a combined follicle-stimulating hormone +human menopausal gonadotropin regime and gonadotropin-releasing hormone (GnRH)-antagonist commenced on stimulation day $6[10,11]$. Estradiol and follicular monitoring via transvaginal ultrasound continued until at least three follicles had maximum diameter of $\geq 17 \mathrm{~mm}$. This threshold was achieved after a 12 day follicular recruitment interval, and 2500 IU hCG+80 u GnRH-agonist (leuprolide) was administered subcutaneous. with oocyte retrieval scheduled 36 hours later. Because PGS was planned, fertilization for all oocytes was exclusively via intracytoplasmic sperm injection (ICSI) [12].

\section{Embryo culture, biopsy, and modified PGS protocol}

Embryos were initially cultured in $10 \mu \mathrm{L}$ microdrops of G1:3 culture medium (Vitrolife, Goteborg, Sweden), and on d3 post-fertilization placed in $20 \mu$ of G2:3 (Vitrolife) to support blastocyst culture. As all embryos in this study had six or more cells by $\mathrm{d} 3$, each was placed in $20 \mu \mathrm{L}$ of G-MOPS medium (Vitrolife) under mineral oil and subjected to biopsy following zona ablation via noncontact laser (ZILOS-tk; Hamilton Thorne Inc., Beverley, MA, USA). Cells from each embryo were removed using micromanipulation and placed immediately in RNAse-DNAse-free polmerase chain reaction (PCR) tubes containing $10 \mu \mathrm{L}$ of PCR-grade water. Following addition of $50 \mu \mathrm{g} / \mathrm{mL}$ Proteinase $\mathrm{K}$ (Roche Biochemicals, Mannheim, Germany) each sample was placed under oil and transferred to our laboratory. DNA assessment used the Illumina Infinium HumanKaryomap-12 DNA Analysis kit. 
Beadchips were read by the iScan array scanner. Data were analyzed with BlueFuse Multi software to determine familial haploblocks. The screening protocol involved a first-round multiplex PCR, simultaneously amplifying the coding region of NLRP7 along with two unlinked fluoroscopically labelled (Cy5.5) microsatellite markers, GA$B R B 3$, and D13S314. The first round PCR reaction was then subject to (1) analysis of NLRP7 genotypes using real-time PCR protocol and allele-specific hybridization probes using the LightCycler system 1.0 (Roche Diagnostics, Manheim, Germany) and (2) microsatellite sizing on a Visible Genetics OpenGene System automatic DNA sequencer with Gene Objects software (Visible Genetics, High Wycombe, UK) to exclude chance contamination, as described previously [13].

\section{Results}

\section{Mutation analysis}

Variants in the NLRP7 gene were annotated following American College of Medical Genetics guidelines for interpretation. A substitution affecting the invariant donor splice site at position c.2810+2T $>G$ of the coding region was identified in the sample obtained from the wife (Figure 1), while no pathogenic or likely pathogenic variants were identified in the husband. This pathogenic variant was shown to alter splicing of intron 9 and result in abnormal retention of the intron, which in turn inserts 54 amino acids in the protein (Figure 2). Of note, this pathogenic variant was previously reported in three patients with recurrent $\mathrm{HM}$, all of Latino-American origin [14]. Our patient was fully informed and counseled about all results when available, and elected to proceed with IVF, PGS, and potential single embryo transfer.

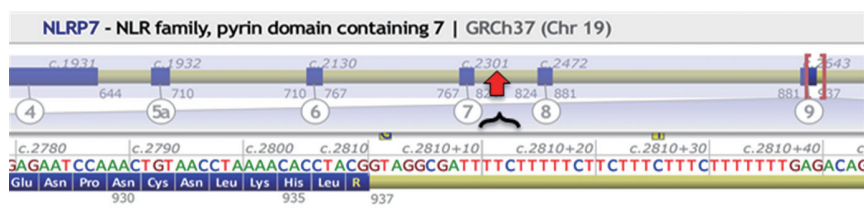

ITCTTAGGTTTGACATTGGATTTTGT GGATGCCATCCGCTAAAAGAAAAAGAAGAAAGAAAGAAAAAAACTCTGTC

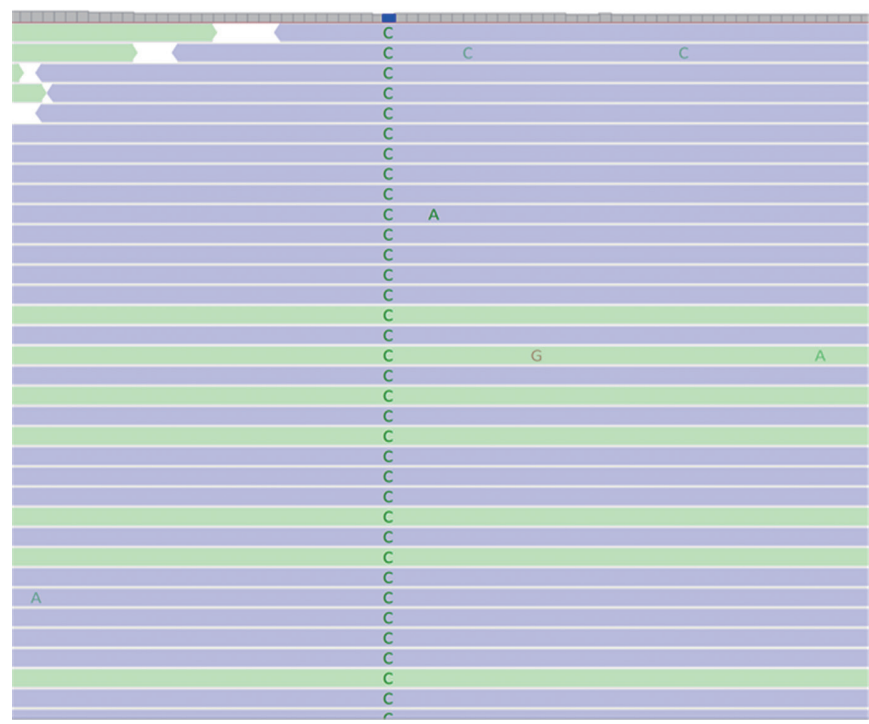

Figure 1. Pathogenic variant in the NLRP7 gene identified in a patient with recurrent hydatidiform mole and global arrest of in vitro fertilization embryos. A substitution affecting the conserved donor splice site at position c. $2810+2 \mathrm{~T}>\mathrm{G}$ of the coding region (black brackets and red arrow), intron 9 of the NLRP7 gene, was identified in the wife. This position had a coverage of $142 X$ in an area of good quality sequence (i.e., further confirmation not required).

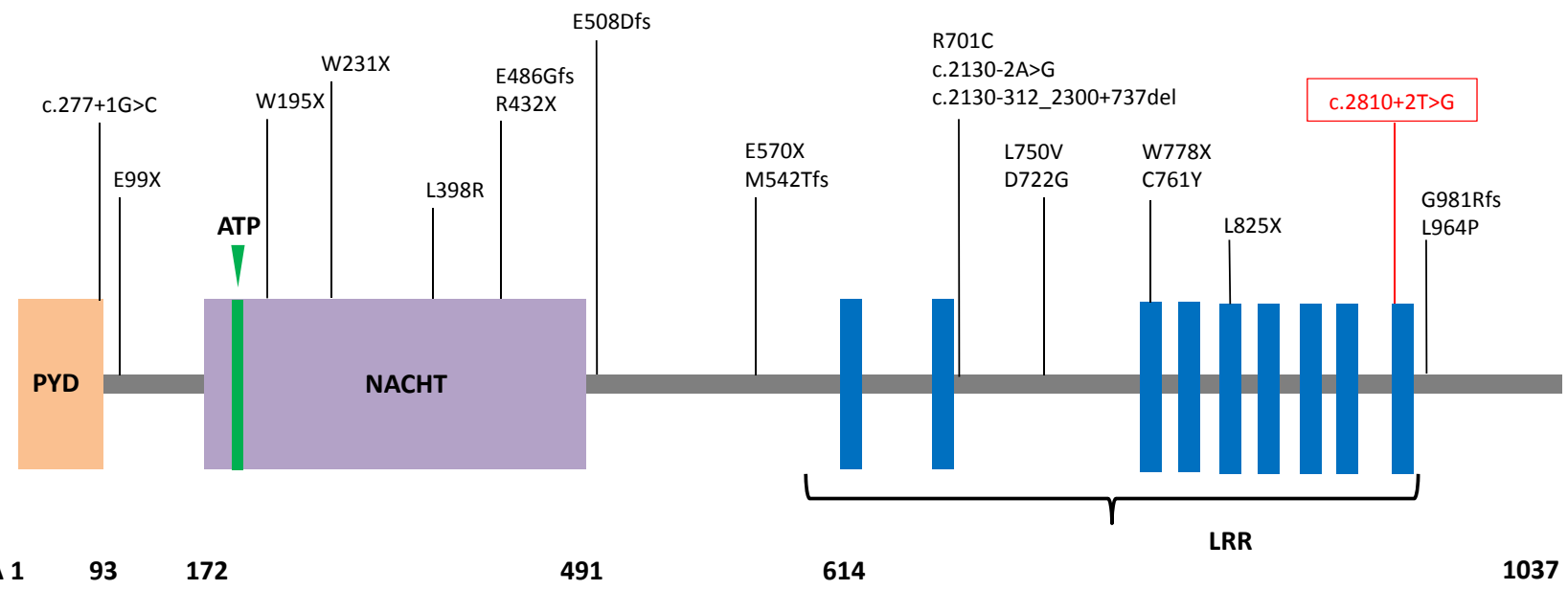

Figure 2. Schematic of NLRP7 protein structure with representative mutations identified in patients with hydatidiform mole. The homozygous mutation present in the index case is shown in red. PYD, pyrin domain; ATP, adenosine 5'-triphosphate binding motif; NACHT, found in NAIP, CIITA, HET-E, and TP1 protein family; LRR, leucine rich region; AA, amino acid number. Mutation nomenclature shown follows Human Genome Variation Society convention. 

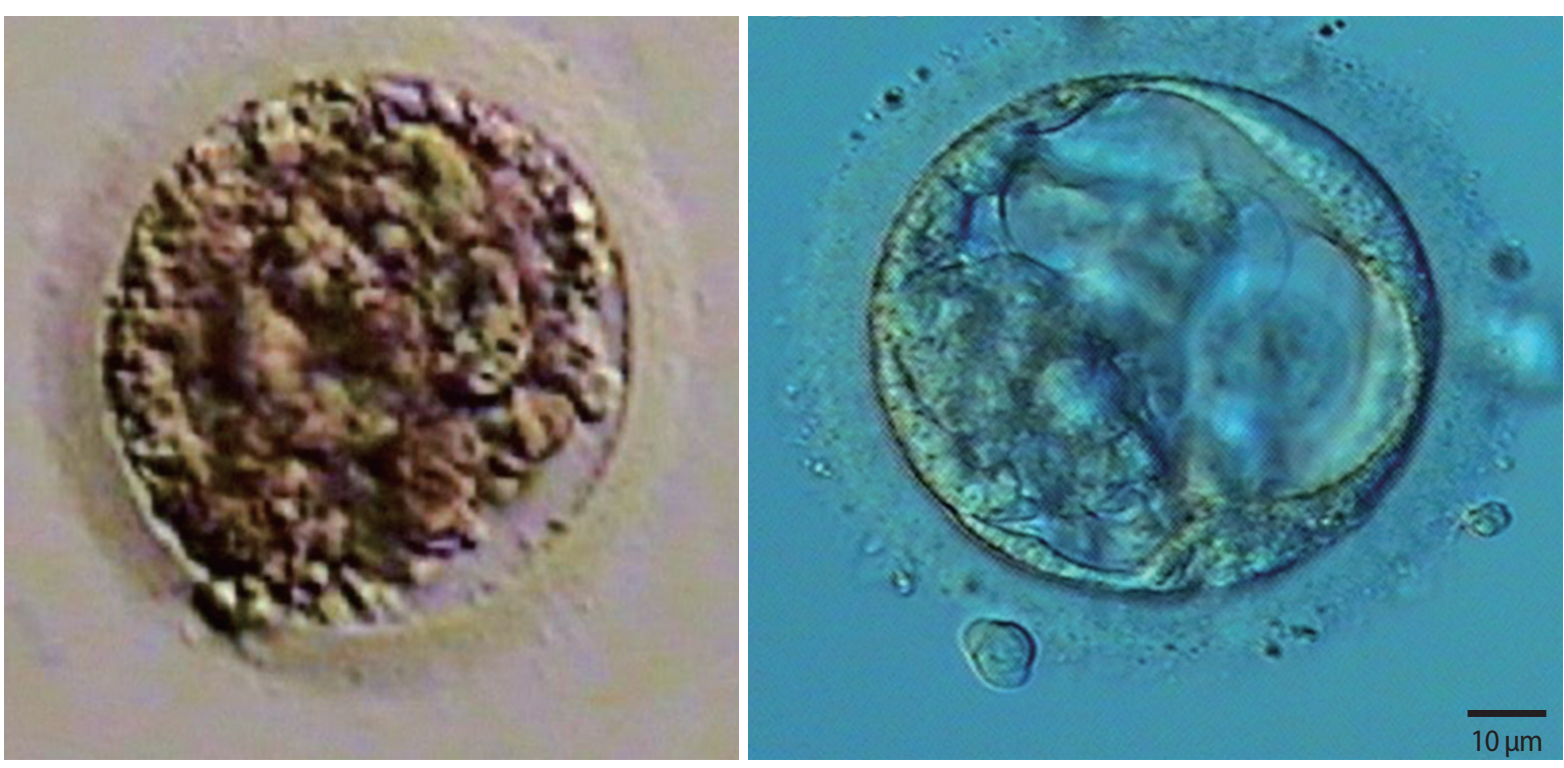

Figure 3. Representative images of embryos derived from a mother with homozygous mutation at c.2810+2T $>G$ and unaffected father. Developmental arrest was observed in all 10 embryos generated after intracytoplasmic sperm injection, with ploidy errors identified in six of 10 embryos.

Table 1. Summary of oocyte status and embryo development during extended in vitro culture

\begin{tabular}{cccccc}
\hline No. & $\begin{array}{c}\text { Oocyte } \\
\text { maturation }\end{array}$ & $24 \mathrm{hr}$ & $72 \mathrm{hr}^{\mathrm{a}}$ & $144 \mathrm{hr}$ & Status \\
\hline 1 & MII & $2 \mathrm{pn}$ & $8 \mathrm{~b} 2 \mathrm{ff}$ & $\mathrm{pm}$ & $\begin{array}{c}10 \text { Embryos } \\
\text { submitted for PGS }\end{array}$ \\
2 & MII & $2 \mathrm{pn}$ & $7 \mathrm{~b} 2 \mathrm{ff}$ & $\mathrm{pm}$ & \\
3 & MII & $2 \mathrm{pn}$ & $7 \mathrm{~b} 2 \mathrm{ff}$ & $\mathrm{pm}$ & \\
4 & MII & $2 \mathrm{pn}$ & $7 \mathrm{~b} 2 \mathrm{ff}$ & - & \\
5 & MII & $2 \mathrm{pn}$ & $7 \mathrm{~b} 2 \mathrm{ff}$ & - & \\
6 & MII & $2 \mathrm{pn}$ & $6 \mathrm{~b} 2 \mathrm{fff}$ & - & \\
7 & MII & $2 \mathrm{pn}$ & $6 \mathrm{~b} 2 \mathrm{fff}$ & - & \\
8 & MII & $2 \mathrm{pn}$ & $5 \mathrm{~b} 2 \mathrm{fff}$ & - & \\
9 & MII & $2 \mathrm{pn}$ & $5 \mathrm{~b} 2 \mathrm{fff}$ & - & \\
10 & MII & $0 \mathrm{pn}$ & $6 \mathrm{~b} 2 \mathrm{f}$ & - & \\
11 & MII & $4 \mathrm{pn}$ & - & - & Arrest after 24 hr, \\
& & & & & not tested \\
12 & Ml & $0 \mathrm{pn}$ & - & - & \\
13 & deg & - & - & - & \\
14 & deg & - & - & - & \\
15 & G & - & - & - & \\
\hline
\end{tabular}

MII, metaphase two; pn, pronuclei; pm, premorula; PGS, preimplantation genetic screening; MI, metaphase one; deg, degenerated; $G$, giant cell (abnormal).

a)Embryo morphological scores assigned according to the system of Gardner and Schoolcraft [15].

\section{IVF response, embryology data, and PGS findings}

Fifteen oocytes were retrieved and nine progressed to 2 pronuclei (pn) stage following ICSI. An additional oocyte (initially designated as $0 \mathrm{pn}$ ) cleaved after 72 hours and joined the others as a provisionally
Table 2. Summary of findings from preimplantation genetic screening from 10 non-viable embryos obtained from IVF where the oocyte source carried a pathogenic (homozygous) variant at NLRP7 (2810 $+2 \mathrm{~T}>\mathrm{G}$ )

\begin{tabular}{ccl}
\hline No. & karyotype data & \multicolumn{1}{c}{ PGS status } \\
\hline 1 & $45, \mathrm{XY} ;$ del $(8 \mathrm{p}),-16$ & Indet \\
2 & $45, \mathrm{XX} ;-8$ & Maternal monosomy \\
3 & $46, \mathrm{XY}$ & Biparental \\
4 & $47, \mathrm{XY} ;+21$ & Maternal trisomy (21) \\
5 & $47, \mathrm{XX} ;+22$ & Maternal trisomy (22) \\
6 & $47, \mathrm{XX} ;+2$ & Maternal trisomy (2) \\
7 & $46, \mathrm{XX}$ & Biparental \\
8 & $45, \mathrm{XX} ;-21$ & Indet \\
9 & $46, \mathrm{XX} ;+9,-19$ & Indet (9); maternal monosomy (19) \\
10 & $\mathrm{C} / \mathrm{a}$ & Complex/indet \\
\hline
\end{tabular}

IVF, in vitro fertilization; PGS, preimplantation genetic screening; del, deletion; Indet, ploidy error of indeterminate parental origin; c/a, complex (>3) chromosomal error.

viable zygote. However, arrest of all embryos was noted between 72 and 144 hours in culture (Figure 3), rendering none suitable for transfer. Progression of embryo development and associated morphology is summarized in Table 1. The 10 non-viable embryos were submitted for molecular diagnosis and biparental chromosomal contributions were identified in all embryos. While ploidy error for some embryos was of indeterminate origin, none was of paternal origin while five were of maternal origin (Table 2). A summary of NGS findings and genetic recombination events observed in the two euploid embryos is presented in Figures 4 and 5. 


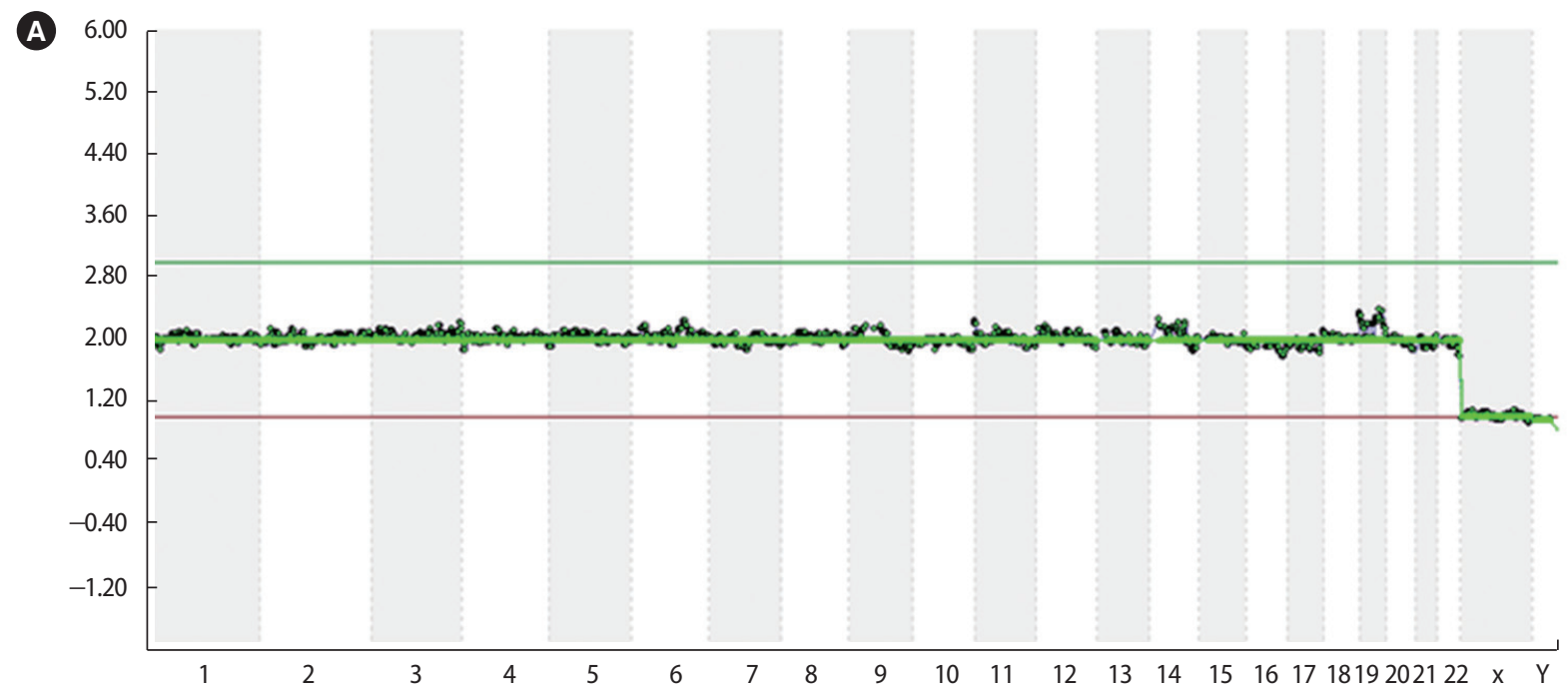

B

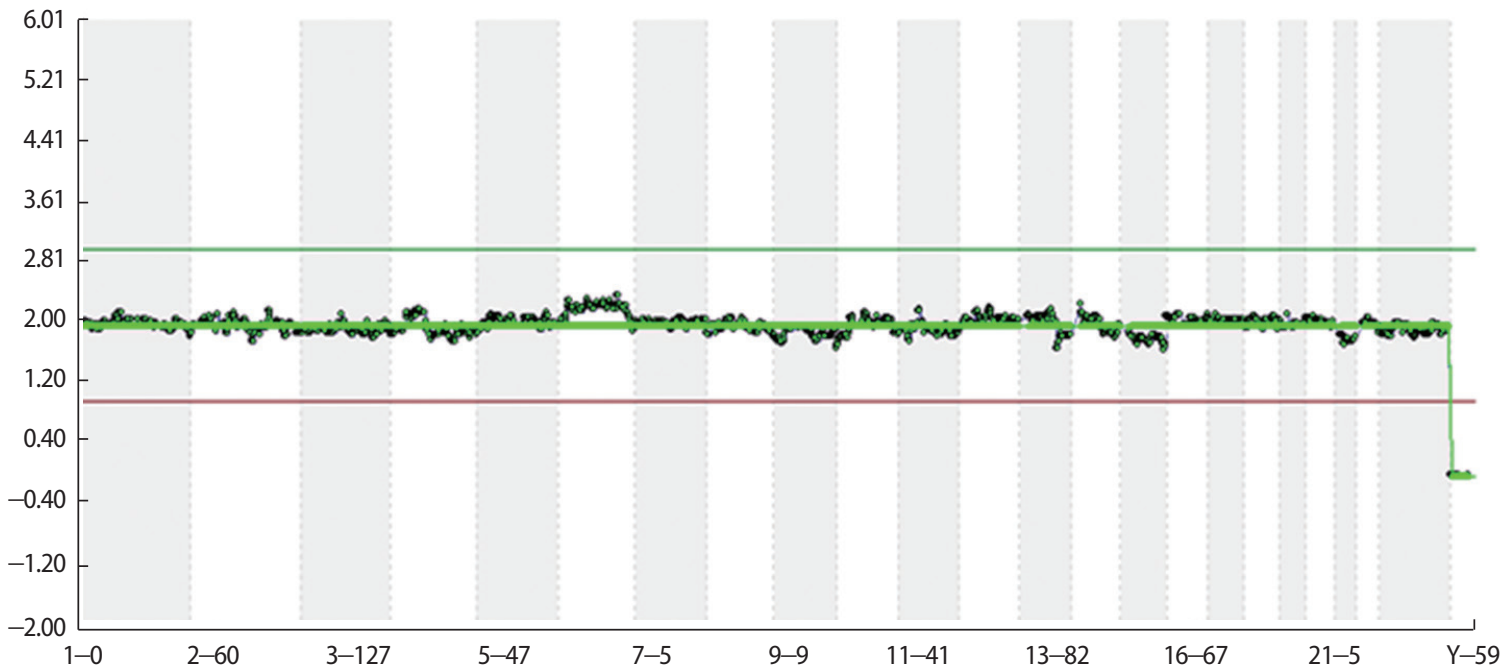

Figure 4. Next generation sequencing data derived from two euploid embryos (sample \#3 (A): 46,XY; sample \#7 (B): 46,XX) obtained from a patient with recurrent hydatidiform mole and homozygous pathogenic variant in $N L R P 7(c .2810+2 T>G)$.

\section{Discussion}

NLRP7 is a maternal-effect gene believed to be required in oocytes to sustain normal development of the early human embryo. Previous research has shown that assisted reproductive technologies incorporating donor oocytes can correct the rare problem of recurrent $\mathrm{HM}$ [6]. Within oocytes, NLRP7 localizes to the cytoskeleton and is abundant in the cortical region [16]. However, the actual consequence of a mutation at NLRP7 as monitored during in vitro embryo culture has not been directly observed and reported until now.

For our patient, her history of five consecutive HM pregnancies implicated a genetic etiology for her condition. As with the previously reported mutation at c.2810, our patient with recurrent HM in California was also of Latino ethnicity, although our case was homozygous for this mutation while the earlier analysis identified a com- pound heterozygous state [14]. While our report is the first to describe development of 2 pn zygotes and abnormal fertilization after ICSI (as well as continued albeit variable development among observed embryos), we can only speculate why some embryos arrested sooner than others. The role of our patient's uterine anomaly in the context of recurrent $\mathrm{HM}$ is probably coincidental; to date, there has only been one published case describing the coexistence of bicornuate uterus and HM [17]. As our patient was known to harbor a homozygous mutation at NLRP7, all embryos generated were expected to inherit one abnormal copy of the gene responsible for HM. With a large enough denominator of screened embryos available for screening, we hypothesized that at least one euploid embryo or blastocyst might enable a transfer and subsequent opportunity for healthy pregnancy given this autosomal recessive disorder. Two euploid embryos were indeed identified for this couple after comprehensive 

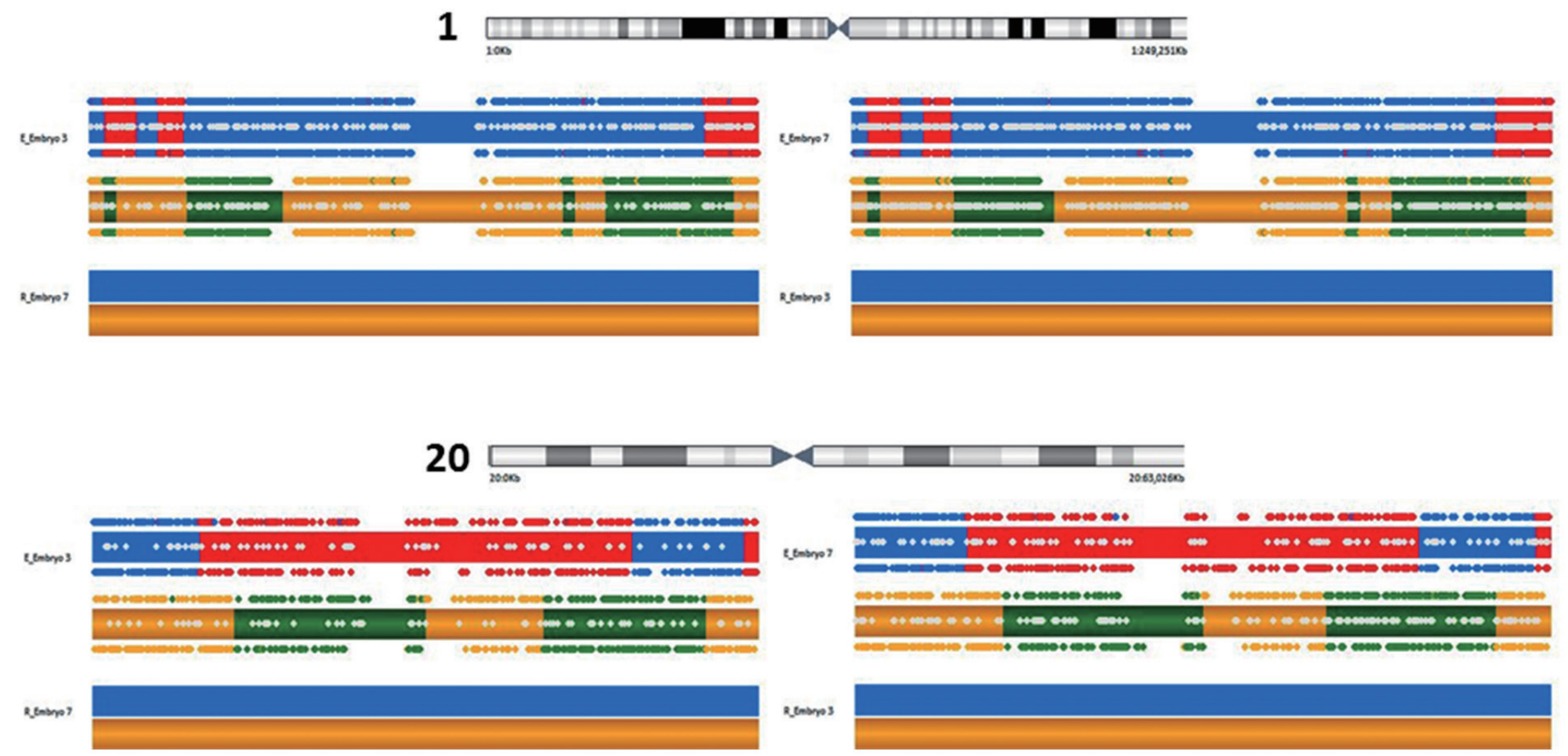

Figure 5. Karyomap and haploblock summary of chromosome 1 (upper) and 20 (lower) as representatives of euploid embryo \#3 and \#7 derived from an in vitro fertilization patient with recurrent hydatidiform mole and homozygous pathogenic variant in NLRP7. Maternally inherited material appears as yellow (shared with reference) and green, while paternally inherited material is shown in blue (shared with reference) and red. Chromosomes are mapped according to predicted phase by BlueFuse software, alternating colors represent recombination events.

chromosomal screening, but unfortunately both embryos arrested in culture and no in utero transfer was possible. This observation raises questions about the reasons behind this developmental collapse.

NLRP7 subcellular localization has been explored using an ex vivostimulated peripheral blood mononuclear cell model, revealing that NLRP7 co-localizes with the Golgi apparatus and the microtubule organizing center. Interestingly, a defect in microtubule and actin filament organization was suggested by Edwards et al. [18] to explain postzygotic abnormalities during in vitro culture of embryos from a patient with recurrent HM. A maternal-effect model has thus been proposed to explain the transcript abundance of NLRP7 which accumulates in developing oocytes and in early preimplantation embryos [19]. Such expression profiles suggest an involvement in the control of maternally-derived epigenetic programing or early developmental events in the zygote that occur ahead of embryonic genome activation. It has also been proposed that a mutation at NLRP7 may interfere with expression of imprinted genes on other chromosomes [20]. Dysfunction of such processes could explain why global embryonic arrest was observed in our case. Thus it may be that the presence of only one copy of this mutation at NLRP7 is sufficient to interrupt normal human embryo development.

It may be inferred that maternal NLRP7 mutations impair cytokine secretion by affecting their trafficking either through the classical Golgi-mediated secretory pathway or by disrupting the microtubule net- work known to play a role in the non-classical pathway of interleukin$1 \beta$ secretion. In contrast, paternal transmission of NLRP7 mutations does not interfere with spermatogenesis, as males homozygous for NLRP7 mutations can father children [21,22]. How such a maternal mutation might manifest in the context of human embryo development has not been directly observed until now. The earlier hypothesis predicting developmental arrest secondary to a NLRP7 mutation is validated by the current case, which graphically highlights the catastrophic disruption of human embryo growth when fundamental cytoskeleton elements are disabled due to this specific mutation.

In contrast to these findings from preimplantation embryos, genotyped molar tissues from pregnant patients with recessive NLRP7 mutations are diploid biparental and do not have aneuploidies. The observed genetic differences between IVF embryos and chorionic villi of HM might be explained by the fact that HM tissues are genotyped with multiplex microsatellite markers, a technique less comprehensive than karyomapping and unable to detect all aneuploidies. Perhaps more crucially, molar tissue is typically sampled during the first trimester and may have been subject to preferential selection or growth of cells without ploidy error, a phenomenon well documented in fetal loss where aneuploidy rates decline with advancing gestational age.

As oocyte donation is being considered by our patient now, her prognosis for a healthy pregnancy with IVF and single embryo transfer is bright. In the future, patients with this NLRP7 mutation should 
be advised about the potential for cycle cancellation due to embryo arrest, and donor egg/embryo IVF or adoption encouraged as preferred management.

\section{Conflict of interest}

No potential conflict of interest relevant to this article was reported.

\section{References}

1. Hoffner L, Surti U. The genetics of gestational trophoblastic disease: a rare complication of pregnancy. Cancer Genet 2012;205:63-77.

2. Judson $\mathrm{H}$, Hayward BE, Sheridan E, Bonthron DT. A global disorder of imprinting in the human female germ line. Nature 2002; 416:539-42.

3. Al-Ghamdi AA. Recurrent hydatidiform mole: a case report of six consecutive molar pregnancies complicated by choriocarcinoma, and review of the literature. J Family Community Med 2011; 18:159-61.

4. Parry DA, Logan CV, Hayward BE, Shires M, Landolsi H, Diggle C, et al. Mutations causing familial biparental hydatidiform mole implicate c6orf221 as a possible regulator of genomic imprinting in the human oocyte. Am J Hum Genet 2011;89:451-8.

5. Dixon PH, Trongwongsa P, Abu-Hayyah S, Ng SH, Akbar SA, Khawaja NP, et al. Mutations in NLRP7 are associated with diploid biparental hydatidiform moles, but not androgenetic complete moles. J Med Genet 2012;49:206-11.

6. Fisher RA, Lavery SA, Carby A, Abu-Hayyeh S, Swingler R, Sebire NJ, et al. What a difference an egg makes. Lancet 2011;378:1974.

7. Deng L, Zhang J, Wu T, Lawrie TA. Combination chemotherapy for primary treatment of high-risk gestational trophoblastic tumour. Cochrane Database Syst Rev 2013;(1):CD005196.

8. Ito Y, Maehara K, Kaneki E, Matsuoka K, Sugahara N, Miyata T, et al. Novel nonsense mutation in the NLRP7 gene associated with recurrent hydatidiform mole. Gynecol Obstet Invest 2016;81:353-8.

9. Sills ES, Alper MM, Walsh AP. Ovarian reserve screening in infertility: practical applications and theoretical directions for research. Eur J Obstet Gynecol Reprod Biol 2009;146:30-6.

10. Sills ES, Schattman GL, Veeck LL, Liu HC, Prasad M, Rosenwaks Z. Characteristics of consecutive in vitro fertilization cycles among patients treated with follicle-stimulating hormone (FSH) and human menopausal gonadotropin versus FSH alone. Fertil Steril 1998;69:831-5.

11. Sills ES, Collins GS, Salem SA, Jones CA, Peck AC, Salem RD. Balancing selected medication costs with total number of daily injections: a preference analysis of $\mathrm{GnRH}$-agonist and antagonist protocols by IVF patients. Reprod Biol Endocrinol 2012;10:67.

12. Palermo G, Joris H, Devroey P, Van Steirteghem AC. Pregnancies after intracytoplasmic injection of single spermatozoon into an oocyte. Lancet 1992;340:17-8.

13. Vrettou C, Traeger-Synodinos J, Tzetis M, Palmer G, Sofocleous C, Kanavakis E. Real-time PCR for single-cell genotyping in sickle cell and thalassemia syndromes as a rapid, accurate, reliable, and widely applicable protocol for preimplantation genetic diagnosis. Hum Mutat 2004;23:513-21.

14. Kou YC, Shao L, Peng HH, Rosetta R, del Gaudio D, Wagner AF, et al. A recurrent intragenic genomic duplication, other novel mutations in NLRP7 and imprinting defects in recurrent biparental hydatidiform moles. Mol Hum Reprod 2008;14:33-40.

15. Gardner DK, Schoolcraft WB. In vitro culture of human blastocysts. In: Jansen R, Mortimer D, editors. Towards reproductive certainty: fertility and genetics beyond 1999. Carnforth: Parthenon Publishing (Lond); 1999. p. 378-88.

16. Messaed C, Akoury E, Djuric U, Zeng J, Saleh M, Gilbert L, et al. NLRP7, a nucleotide oligomerization domain-like receptor protein, is required for normal cytokine secretion and co-localizes with Golgi and the microtubule-organizing center. J Biol Chem 2011;286:43313-23.

17. Krishnamoorthy K, Gerkowicz S, Verma U. Viable intrauterine pregnancy and coexisting molar pregnancy in a bicornuate uterus: a rare presentation. J Clin Imaging Sci 2016;6:26.

18. Edwards R, Crow J, Dale S, Macnamee M, Hartshorne G, Brinsden P. Preimplantation diagnosis and recurrent hydatidiform mole. Lancet 1990;335:1030-1.

19. Akoury E, Zhang L, Ao A, Slim R. NLRP7 and KHDC3L, the two maternal-effect proteins responsible for recurrent hydatidiform moles, co-localize to the oocyte cytoskeleton. Hum Reprod 2015;30:159-69.

20. Fisher RA, Hodges MD, Rees HC, Sebire NJ, Seckl MJ, Newlands $\mathrm{ES}$, et al. The maternally transcribed gene p57(KIP2) (CDNK1C) is abnormally expressed in both androgenetic and biparental complete hydatidiform moles. Hum Mol Genet 2002;11:3267-72.

21. Wang CM, Dixon PH, Decordova S, Hodges MD, Sebire NJ, Ozalp $\mathrm{S}$, et al. Identification of 13 novel NLRP7 mutations in 20 families with recurrent hydatidiform mole; missense mutations cluster in the leucine-rich region. J Med Genet 2009;46:569-75.

22. Sanchez-Delgado M, Martin-Trujillo A, Tayama C, Vidal E, Esteller $\mathrm{M}$, Iglesias-Platas I, et al. Absence of maternal methylation in biparental hydatidiform moles from women with NLRP7 maternal-effect mutations reveals widespread placenta-specific imprinting. PLoS Genet 2015;11:e1005644. 